\title{
Evaluación de la distribución de esfuerzos de una restauración inmediata implantosoportada en tres fases de cicatrización. Análisis de elementos finitos
}

Evaluation of the distribution of efforts of an immediate restoration supported in three phases of healing. Finite element analysis

Avaliação da distribuição de esforços de uma restauração imediata implantossuportada em três fases de cicatrização. Análise de elementos finitos

\section{Mauricio Alejandro Rodríguez López ${ }^{1}$ Samuel David Giraldo Gómez ${ }^{2}$ Federico Latorre Correa ${ }^{3}$ Junes Abdul Villarraga Ossa ${ }^{4}$ Juan Gustavo Diosa Peña ${ }^{5}$}

Artículo de investigación. https://doi.org/10.16925/2357-4607.2019.01.06

1 Facultad de Odontología, Universidad Cooperativa de Colombia https://orcid.org/0000-0002-3470-591X

2 Facultad de Odontología, Universidad Cooperativa de Colombia https://orcid.org/0000-0001-6645-8792

Correo electrónico: samuel.giraldo@campusucc.edu.co

3 Facultad de Odontología, Universidad de Antioquia. https://orcid.org/0000-0003-4432-3584

4 Facultad de Ingeniería, Universidad de Antioquia. https://orcid.org/0000-0002-7893-5362

5 Facultad de Ingeniería de Materiales, Universidad de Antioquia. https://orcid.org/0000-0002-8064-2470 
Recibido: 16 de julio de 2017

Aprobado: 20 de junio de 2018

Publicado: 20 de marzo de 2019

Cómo citar este artículo: Rodríguez-Lopez MA, Giraldo-Gómez SD, Latorre-Correa F, Villarraga-Ossa JA, Diosa-Peña JG. Evaluación de la distribución de esfuerzos de una restauración inmediata implantosoportada en tres fases de cicatrización. Análisis de elementos finitos. Rev Nac Odontol. 2019;15(28):1-15. doi: https://doi.org/10.16925/2357-4607.2019.01.06

\section{Resumen}

Introducción: evaluar la distribución de esfuerzos von Mises en restauraciones implanto soportadas, con un pilar temporal de Peek y uno de titanio, en tres etapas de cicatrización (día cero, 1.5 meses y 3 meses). Estas evaluaciones fueron $r$ mef).

Métodos: se modeló un implante Tapered Screw-Vent ${ }^{\circledR}$ (ref. TSVB10 Zimmer Dental) de $13 \mathrm{~mm}$ de longitud por $3.7 \mathrm{~mm}$ diámetro con una plataforma de $3.5 \mathrm{~mm}$, un pilar de Peek, un pilar de titanio, un tornillo, una corona de Pmmade un incisivo central superior, un hueso cortical y esponjoso con diferentes densidades dependiendo del estadio de cicatrización; se utilizó el Software cad de Solid Works 2010, se procesó y analizó a través del Software ansys versión 14. Se evaluó la distribución de esfuerzos von Mises, aplicando cargas en sentido oblicuo con una magnitud de $200 \mathrm{~N}$.

Resultados: la concentración de stress en el hueso esponjoso apical es 10 veces mayor en el día cero que en los demás momentos de cicatrización. Los modelos de pilares en Peek en el momento 1.5 y 3 meses mostraron casi dos veces mayores esfuerzos en el implante que los modelos en titanio; se observaron valores similares von Mises al comparar el momento 1.5 y 3 meses.

Conclusiones: en el día cero de cicatrización se concentra la mayor cantidad de esfuerzo en la porción apical del hueso esponjoso, los pilares de Peek trasmiten mayor esfuerzo al tornillo del implante, la formación de hueso crestal ayuda a una mejor distribución de los esfuerzos en el sistema.

Palabras clave: Pilar Peek, pilar titanio, implante dental, análisis de elementos

distribución de esfuerzos.

\section{Summary}

Introduction: to evaluate the distribution of von Mises stress in implant-supported restorations, with a temporary pillar of Peek and one of titanium, in three stages of healing (zero day, 1.5 months and 3 months). These evaluations were carried out $b$ fem) method.

Methods: A Tapered Screw-Vent ${ }^{\circledR}$ implant (ref .: TSVB10 Zimmer Dental) of $13 \mathrm{~mm}$ length by $3.7 \mathrm{~mm}$ diameter was modeled with a $3.5 \mathrm{~mm}$ platform, a Peek abutment, a titanium abutment, a screw, a crown Pmma of an upper central incisor, a cortical and spongy bone with different densities depending on the stage of healing; the Solid Works 2010 cad Software was used, processed and analyzed through the ansys Software version 14. The von Mises stress distribution was evaluated, applying oblique loads with a magnitude of $200 \mathrm{~N}$.

Results: the concentration of stress in the apical spongy bone is 10 times greater on day zero than in the other moments of healing. The models of abutments in peek at the time 1.5 and 3 months showed almost two times greater efforts in the implant than the models in titanium; similar values were observed von Mises when comparing the moment 1.5 and 3 months.

Conclusions: on the zero day of healing the greatest amount of effort is concentrated in the apical portion of the cancellous bone, the Peek pillars transmit more effort to the implant screw, the crestal bone formation helps a better distribution of the stress in the system.

Keywords: Peek ess distribution. 
Mauricio Alejandro Rodríguez López, Samuel David Giraldo Gómez, Federico Latorre Correa, 3 Junes Abdul Villarraga Ossa, Juan Gustavo Diosa Peña

\section{Resumo}

Introdução: avaliar a distribuição do estresse de von Mises em restaurações implantossuportadas, com um pilar temporário de Peek e um de titânio, em três estágios de cicatrização (zero dia, 1,5 meses e 3 meses). Essas avaliações foram $r$

mef).

Métodos: Um implante Tapered Screw-Vent ${ }^{\circledR}$ (ref.: TSVB10 Zimmer Dental) de $13 \mathrm{~mm}$ de comprimento por 3,7 mm de diâmetro foi modelado com uma plataforma de 3,5 mm, um pilar Peek, um pilar de titânio, um parafuso, uma coroa Pmma de um incisivo central superior, um osso cortical e esponjoso com diferentes densidades dependendo do estágio de cura; 0 software cad Solid Works 2010 foi utilizado, processado e analisado através da versão 14 do software ansys . A distribuição de tensão de von Mises foi avaliada, aplicando cargas oblíquas com magnitude de $200 \mathrm{~N}$.

Resultados: a concentração de estresse no osso esponjoso apical é 10 vezes maior no dia zero do que nos outros momentos de cicatrização. Os modelos de abutments em Peek na época de 1,5 e 3 meses mostraram esforços quase duas vezes maiores no implante do que os modelos em titânio; Valores semelhantes foram observados em von Mises quando comparados os momentos 1,5 e 3 meses.

Conclusões: no dia zero de cicatrização a maior quantidade de esforço está concentrada na porção apical do osso esponjoso, os pilares do Peek transmitem mais esforço ao parafuso do implante, a formação do osso crestal ajuda a uma melhor distribuição do estresse no sistema

Palavras-chave: Pilar Peek, pilar de titânio, implante dentário, análise de elementos tensões.

distribuição de

\section{Introducción}

En la planeación de la rehabilitación fija sobre implantes oseointegrados hay que tener en cuenta factores como: densidad ósea, propiedades mecánicas que se presentan en la interfase implante-hueso, magnitud y dirección de las fuerzas oclusales, la distribución de los implantes, entre otros $(1,2)$.

La carga o rehabilitación inmediata es la colocación de una corona provisional inmediatamente después de la inserción del implante, esta se ha podido realizar gracias a los cambios geométricos y superficiales de los implantes mejorando la estabilidad primaria y acelerando el proceso de oseointegración. Se pueden tomar diferentes posturas en cuanto a si se realiza o no carga inmediata. La toma de esta decisión se basa en diferentes criterios que van desde el tipo de implante que se colocó, hasta las diferentes condiciones que se pueden encontrar en el paciente; una de estas es la cantidad de hueso disponible (amplitud, espesor y altura) y la calidad o densidad (hueso tipo I, II, III O IV).(3).

La distribución de esfuerzos en los componentes de un implante puede variar por dos factores: a nivel de la prótesis (materiales de restauración, diseño protésico, pilares, patrón oclusal y distribución de los implantes) y de los implantes (diseño, superficie, diámetro y longitud); los cuales a través de la restauración van a recibir las fuerzas oclusales $(4,5)$. Los esfuerzos en un implante deben evitar la fatiga del material y su posible fractura, una concentración de esfuerzos elevados puede producir pérdida 
4 Evaluación de la distribución de esfuerzos de una restauración inmediata implantosoportada en tres fases de cicatrización. Análisis de elementos finitos

de hueso marginal, fractura de la cerámica de recubrimiento, aflojamiento o fractura del tornillo de fijación y fractura del implante $(6,7)$.

Los pilares para provisionalizar sobre implantes están disponibles en dos materiales, uno es el titanio y el otro es el Poliéter-Éter-Cetona (PEEK). El titanio es reconocido por su alto módulo de elasticidad y excelente biocompatibilidad, sin embargo, el color gris puede significar problemas estéticos (8). El PEEK es un material termoplástico fuerte producido a partir de poliéter éter cetona. Debido a su alta resistencia mecánica y durabilidad, buenas características eléctricas y resistencia a la hidrólisis, ha sido utilizado ampliamente en la industria aeroespacial, automotriz, química, electrónica y las industrias de alimentos y bebidas (9). El uso de los pilares en PEEK cada vez toma más fuerza, estos están indicados para procedimientos de provisionalización, solucionan los problemas estéticos que se originan por el uso de pilares en titanio, también por sus propiedades mecánicas las cuales hacen que se transfieran menos cargas al implante, factor importante en situaciones en donde se realiza carga o restauración inmediata en implantes post exodoncia. Koutouzis realizó un estudio clínico aleatorizado sugiriendo que no hay diferencia significativa en la reabsorción ósea entre el uso de pilares en titanio y PEEK (10). Sin embargo, no se encuentran estudios comparativos de la distribución de esfuerzos en los componentes de un sistema de implantes en el momento de usar un pilar en PEEK y otro en titanio, ni donde se evalúe en los diferentes tiempos de cicatrización.

Es importante evaluar la distribución de esfuerzos que se generan en todos los componentes del implante, la variación de estas fuerzas al utilizar un pilar plástico o uno en titanio y cómo el hueso puede reaccionar en diferentes estadios de oseointegración. Para evaluar estos esfuerzos se usará el método numérico de los elementos finitos (MEF); este tiene importancia para la solución de problemas en diferentes ámbitos como lo son la ingeniería, física, medicina, odontología y otras ciencias, estas permiten resolver problemas que por medios matemáticos tradicionales no se podían realizar. El MEF permite realizar un modelo matemático de cálculo del sistema real, más fácil y económico de modificar que un prototipo. Sin embargo, no deja de ser un método aproximado de cálculo debido a las hipótesis básicas del método $(11,12)$. 
Mauricio Alejandro Rodríguez López, Samuel David Giraldo Gómez, Federico Latorre Correa, 5 Junes Abdul Villarraga Ossa, Juan Gustavo Diosa Peña

\section{Materiales y métodos}

Para el modelo geométrico tridimensional, se utilizará el software cad de SolidWorks (SolidWorks, SolidWorks Corp., Concord, MA, USA). SolidWorks es un programa de diseño asistido por computador para modelado mecánico que corre bajo el sistema operativo Microsoft Windows y es desarrollado en la actualidad por SolidWorks Corp., una subsidiaria de Dassault Systèmes (Suresnes, Francia).

Se decide trabajar con la casa comercial Zimmer® debido a que el grupo de investigación tiene modelado el implante y pilar, estos se han estado refinando y mejorando con los años, lo que facilito el montaje de todo el modelo a estudiar.

El modelo se compondrá de un implante Tapered Screw-Vent Zimmer ${ }^{\circledR}$, de un pilar temporal plastico Zimmer ${ }^{\circledR}$, de pilar de titanio Zimmer ${ }^{\circledR}$, Corona en PMMA, hueso cortical, hueso esponjoso, con las siguientes características cada uno:

- Implante Tapered Screw-Vent Zimmer ${ }^{\circledR}$ : diámetro de $4.7 \mathrm{~mm}$, plataforma de $4.5 \mathrm{~mm}$, largo $13 \mathrm{~mm}$, conexión interna hexagonal.

- Pilar de temporal plastico Zimmer ${ }^{\circledR}$ : precontorneado, recto, conexión interna hexagonal, diámetro de $4.5 \mathrm{~mm}$, perfil de emergencia de 5,5mm, altura del margen vestibular 1,0 mm, referencia número: PT451S.

- Pilar en titanio Zimmer ${ }^{\circledR}$ : precontorneado, recto, conexión interna hexagonal, diámetro de $4.5 \mathrm{~mm}$, perfil de emergencia de $4,5 \mathrm{~mm}$, altura del margen vestibular 1,0 mm, referencia número: ZOA441S.

- $\quad$ Casa fabricante: Zimmer ${ }^{\circledR}$ Dental 1900 Aston Avenue Carlsbad, CA 92008-7308 USA.

El hueso cortical se modelará con un espesor de 1mm; para el hueso esponjoso se realizará un modelo tridimensional de los tres diferentes tiempos de oseointegración de la siguiente forma: en los tres modelos a estudiar, el implante estará en contacto con hueso tipo II en el 50 \% de su superficie apical; en el momento cero, la otra mitad del implante no tendrá ningún valor pues clínicamente encontraríamos un coágulo, el cual no aporta mecánicamente al implante (figura 1). En el mes 1.5, se modelará un implante en el cual la mitad crestal estará en contacto con hueso tipo IV simulando un hueso inmaduro en proceso de cicatrización; en el modelo que simulará los tres meses, el 50 \% crestal tendrá un hueso tipo III, simulando un hueso mucho más maduro y con características mecánicas diferentes (figura 2). 
6 Evaluación de la distribución de esfuerzos de una restauración inmediata implantosoportada en tres fases de cicatrización. Análisis de elementos finitos

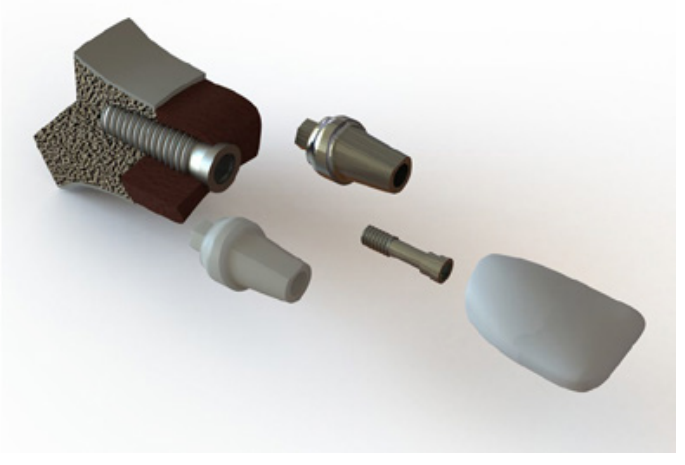

Figura 1. Modelado día cero

Fuente: elaboración propia

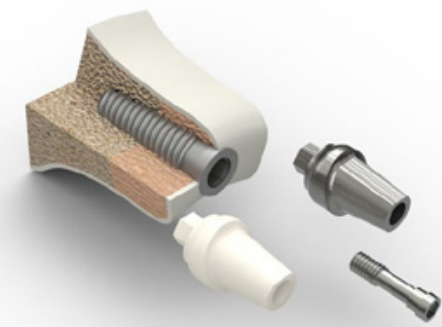

Figura 2. 1.5 y 3 meses

Fuente: elaboración propia.

Es importante tener en cuenta que, desde el punto de vista mecánico, el hueso es considerado anisotrópico porque muestra distintas propiedades mecánicas dependiendo de la dirección desde la cual sean medidas. Sin embargo, por la complejidad de la modelación de una estructura con dichas características a través del método de elementos finitos, puede ser aplicable una aproximación bajo el concepto de isotropía transversal, teniendo en cuenta que el módulo elástico del hueso medido en ciertos ejes posee valores similares (13).

Estudios mecanobiológicos de las etapas de cicatrización han sido mencionadas por Vanegas-Acosta y Sanz, y son referencias que el grupo de investigación del presente artículo ha tenido en cuenta para este modelo $(14,15)$.

Se escogió un implante para un incisivo central superior porque, según los reportes de la literatura, es el más utilizado por razones estéticas, y en este sentido 
Mauricio Alejandro Rodríguez López, Samuel David Giraldo Gómez, Federico Latorre Correa, 7 Junes Abdul Villarraga Ossa, Juan Gustavo Diosa Peña

es importante tomar en cuenta la distribución de esfuerzos. El tipo de superficie del implante, el diámetro y la conexión del pilar modelados son los más utilizados para la rehabilitación oral en el sector anterior (16). Todas las estructuras se modelaron de forma individual, tomando en cuenta un diseño preestablecido. En los modelos no fue tomada en cuenta la encía asumiendo que no tiene un efecto significativo en cuanto a la distribución de esfuerzos.

La condición de precarga en el tornillo se realizó mediante la aplicación de una carga térmica empleando una fórmula matemática $(T=K F d)$ sobre las roscas del tornillo, donde $\mathrm{T}$ es igual al torque, $\mathrm{K}$ es igual a 0,2, F es la fuerza axial y d es igual al diámetro del tornillo (17). Esa contracción térmica uniaxial acorta el área entre las roscas, crea la precarga en el tornillo y genera una fuerza entre éste y el implante, sin disminuir el diámetro original del implante. La cantidad de torque del tornillo fue de 30 Ncm según lo recomendado por la casa fabricante (18).

Los modelos geométricos con las diferentes características se describen en la tabla 1.

Tabla 1. Modelos geométricos y características.

\begin{tabular}{|c|c|c|}
\hline Modelo 1 & Pilar Titanio & \multirow{2}{*}{ Día cero } \\
\hline Modelo 2 & Pilar PEEK & \\
\hline Modelo 3 & Pilar Titanio & \multirow{2}{*}{1.5 meses } \\
\hline Modelo 4 & Pilar PEEK & \\
\hline Modelo 5 & Pilar Titanio & \multirow{2}{*}{3 meses } \\
\hline Modelo 6 & Pilar PEEK & \\
\hline
\end{tabular}

Fuente: elaboración propia

Una vez obtenido el modelo tridimensional se realizó un análisis elástico lineal y no lineal para elaborar una malla sólida, a través de un mallado estándar, donde se utilizaron elementos tetraédricos con el fin de obtener una mejor aproximación de la geometría de las partes. De esta manera se obtiene una malla tridimensional de elementos finitos de los componentes que conforman el modelo (figura 3 y tabla 2). 

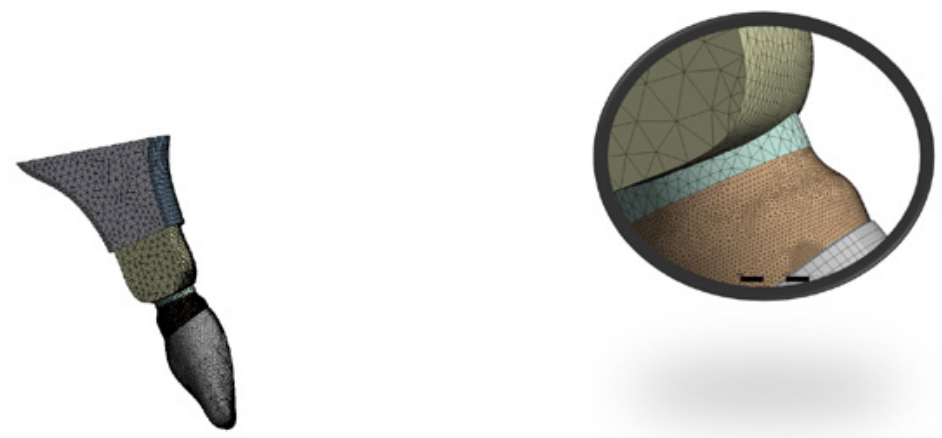

Figura 3. Malla sólida tetraédrica de las estructuras.

Fuente: elaboración propia

Tabla 2. Detalles de la malla del modelo.

\begin{tabular}{ll}
\hline \multicolumn{1}{c}{ CARACTERÍSTICAS DE LA MALLA } & \multicolumn{1}{c}{ MODELO } \\
\hline Tipo de malla & Malla sólida tetraédrica \\
\hline Tamaño de elementos & $0,75 \mathrm{~mm}$ \\
\hline Número total de nodos & 3442891 \\
\hline Número total de elementos & 2335134 \\
\hline Calidad promedio elementos & 0.822 \\
\hline Desviación estándar & 0,116 \\
\hline
\end{tabular}

Fuente: elaboración propia

Al validar las características de la malla se observó un número elevado debido a la calidad promedio de los elementos (0.822). Para mejorar la precisión se utilizó el método adaptativo conocido como método $\mathrm{H}$, el cual consiste en realizar un refinamiento en el tamaño de la malla en los lugares de mayor interés para el estudio. En este caso, la zona en donde ocurren los mayores desplazamientos o esfuerzos.

Para obtener la distribución de esfuerzos mediante el modelo se utilizarán las constantes elásticas, (modelo elástico de Young: propiedad de los materiales que equivale a la pendiente de la curva de esfuerzo contra la deformación en el rango elástico; la razón de Poisson que es una constante elástica de los materiales que indica la relación entre la deformación axial y lateral; y límite de fluencia que es la deformación irrecuperable o irreversible para cada uno de los elementos que se van a analizar). Las propiedades mecánicas de los materiales a usar en este estudio se muestran en la tabla 3. 
Mauricio Alejandro Rodríguez López, Samuel David Giraldo Gómez, Federico Latorre Correa, 9 Junes Abdul Villarraga Ossa, Juan Gustavo Diosa Peña

Tabla 3. Propiedades mecánicas de las estructuras y materiales modelados.

\begin{tabular}{|c|c|c|c|c|c|c|c|c|c|c|}
\hline \multirow[t]{2}{*}{ MATERIAL } & \multicolumn{3}{|c|}{$\begin{array}{l}\text { Limite Elástico } \\
\text { E (MPa) }\end{array}$} & \multicolumn{3}{|c|}{$\begin{array}{l}\text { Coeficiente de } \\
\text { Poisson - V }\end{array}$} & \multicolumn{3}{|c|}{$\begin{array}{c}\text { Límite de } \\
\text { Cizalla } \\
\text { G (MPa) }\end{array}$} & \multirow[t]{2}{*}{$\begin{array}{c}\text { Límite } \\
\text { de } \\
\text { fluencia }\end{array}$} \\
\hline & $\mathbf{X}$ & $\mathbf{Y}$ & $\mathbf{Z}$ & $\mathbf{X Y}$ & $\mathbf{X Z}$ & $\mathbf{Y Z}$ & $\mathbf{X Y}$ & $\mathbf{X Z}$ & $\mathbf{Y Z}$ & \\
\hline Hueso cortical (13) & 12.600 & 12.600 & 19.400 & 0,3 & 0,253 & 0,253 & 4850 & 5700 & 5700 & $60-120$ \\
\hline Hueso esponjoso tipo II (13) & 210 & 1.148 & 1.148 & 0,05 & 0,32 & 0,01 & 68 & 68 & 434 & $60-120$ \\
\hline Hueso esponjoso tipo III (13) & 42 & 230 & 230 & 0,05 & 0,32 & 0,01 & 14 & 14 & 87 & $60-120$ \\
\hline Hueso esponjoso tipo IV (13) & 21 & 115 & 115 & 0,05 & 0,32 & 0,01 & 7 & 7 & 43,5 & $60-120$ \\
\hline $\begin{array}{l}\text { Implante de titanio } \\
(6,7,11,12,19)\end{array}$ & \multicolumn{3}{|l|}{110.000} & \multicolumn{3}{|l|}{0,33} & & & & 800 \\
\hline $\begin{array}{l}\text { Tornillo del pilar titanio } \\
(6,7,11,12,19)\end{array}$ & \multicolumn{3}{|l|}{110000} & \multicolumn{3}{|l|}{0,33} & & & & 800 \\
\hline Pilar de Peek $(19,20)$ & \multicolumn{3}{|l|}{4300} & \multicolumn{3}{|l|}{0,37} & & & & 172 \\
\hline $\begin{array}{l}\text { Pilar de titanio } \\
(6,7,11,12,19)\end{array}$ & \multicolumn{3}{|l|}{110000} & \multicolumn{3}{|l|}{0,33} & & & & 800 \\
\hline $\begin{array}{l}\text { Corona provisional en PMMA } \\
(13,21)\end{array}$ & \multicolumn{3}{|l|}{3000} & \multicolumn{3}{|l|}{0.38} & & & & 72 \\
\hline
\end{tabular}

Fuente: elaboración propia

Una vez obtenida la modelación se realizó un estudio comparativo de los seis modelos analizando los esfuerzos von Mises para todo el sistema, este se llevó a cabo a través del Software ANSYS versión 14; sometiéndose a una carga de 200 N que corresponde a la carga oclusal normal reportada por la literatura para el sector anterior (22), con un vector de fuerza oblicuo a una angulación de $45^{\circ}$ sobre la cara palatina del diente rehabilitado, en un área de $4 \mathrm{~mm}$ (2). Este análisis permitió valorar el comportamiento de las diferentes estructuras modeladas (figura 4).

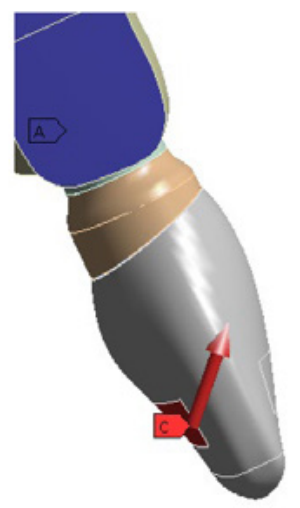

Figura 4. Dirección y ubicación de las cargas. Área en rojo. Fuente: elaboración propia 
Evaluación de la distribución de esfuerzos de una restauración inmediata implantosoportada en tres fases de cicatrización. Análisis de elementos finitos

\section{Resultados}

En la tabla 4 se presenta un resumen de los esfuerzos von Mises para cada componente del sistema de implantes en los seis modelados diferentes.

Tabla 4. Valores en MPa de los esfuerzos máximos von Mises en cada estructura

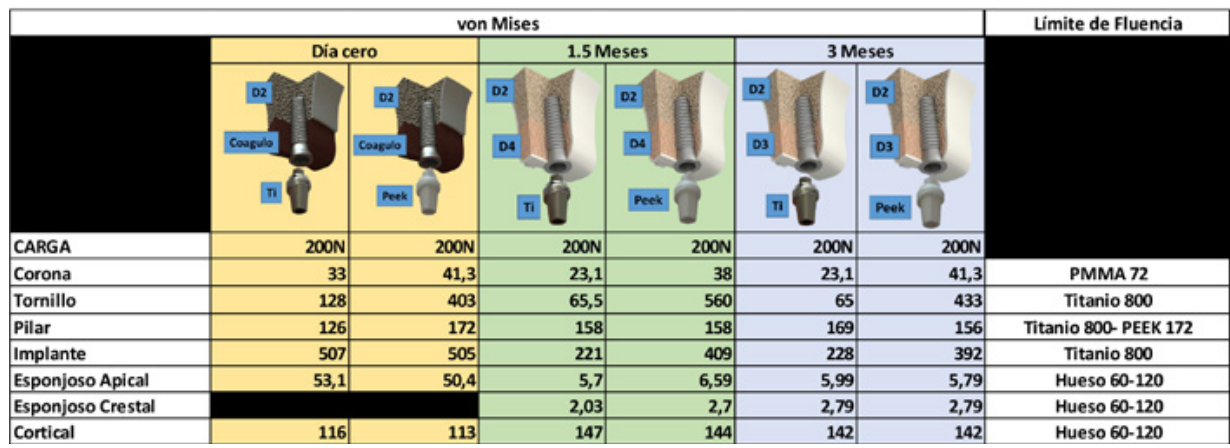

$\mathrm{N}$ : Newtons.

Fuente: elaboración propia

Los resultados de la distribución de esfuerzos en los sistemas tanto en pilares de titanio como PEEK, se observan en las tablas 5, 6 y 7, en cada uno de los momentos de cicatrización: día cero, 1.5 meses y 3 meses respectivamente.

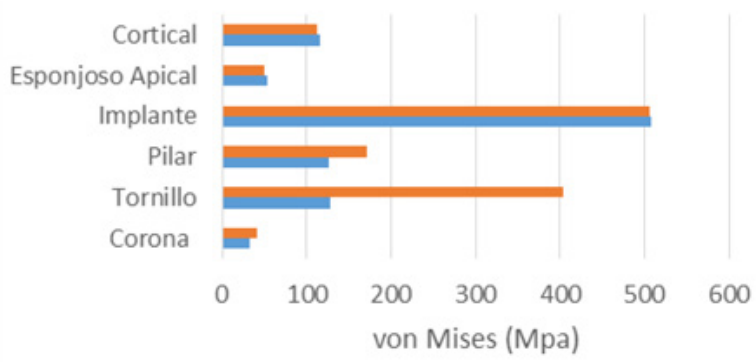

a Pilar en PEEK a Pilar en titanio

Figura 5. Día cero.

Fuente: elaboración propia 
Mauricio Alejandro Rodríguez López, Samuel David Giraldo Gómez, Federico Latorre Correa, 11 Junes Abdul Villarraga Ossa, Juan Gustavo Diosa Peña

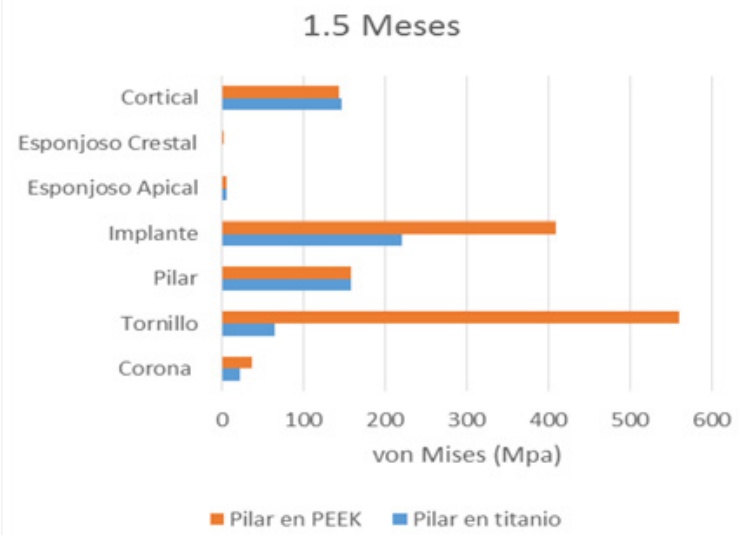

Figura 6. 1.5 Meses.

Fuente: elaboración propia

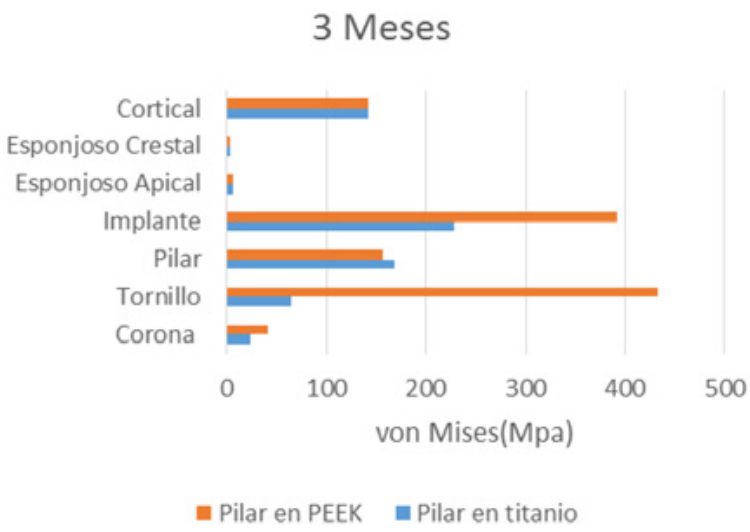

Figura 7. 3 Meses.

Fuente: elaboración propia

\section{Discusión}

Al ser aplicada la carga se observó tanto en los modelados de pilares en titanio como en los de PEEK, una concentración de stress en el hueso esponjoso apical de 10 veces mayor en el día cero que en los demás momentos de cicatrización; en el día cero el implante sólo tiene contacto con el hueso en la porción apical, la porción crestal es un coagulo que no aporta a la distribución de esfuerzos en el sistema, Sanz-Sánchez et al. (23) llegaron a la conclusión de que las fuerzas oclusales impactan directamente en el hueso del implante post-exodoncia y, por lo tanto, los pacientes pueden ser más 
susceptibles al fracaso del tratamiento. Esposito et al. (24) realizaron un metaanálisis donde no encontraron evidencia suficiente para determinar la influencia de la carga oclusal en la supervivencia del implante.

La aparición del hueso esponjoso crestal en los modelos de 1.5 y 3 meses, disminuyó las fuerzas sobre el hueso esponjoso apical que se observó en el día cero. E contacto de hueso que se da en la porción crestal del implante, independiente de la densidad ósea, ayudó a distribuir mejor los esfuerzos que se observaba en la porción apical.

En los modelos con pilares en titanio se observó en el tornillo y en el implante una concentración de fuerzas 2 y 2.5 veces mayor respectivamente en el día cero que en los demás momentos de cicatrización, también al evaluar los componentes del sistema de pilares en titanio con los de PEEK en el día cero, se observa que el esfuerzo se transmite más al tornillo de los pilares de PEEK, esto concuerda con el estudio in-vitro que realizó Neumann E. et al. (25), donde estudió la resistencia del tornillo en tres pilares diferentes, en PEEK, PEEK reforzado con carbono y en titanio, encontrando que el pilar de titanio le ofrece al tornillo una resistencia a la fractura 3,4 veces mayor que los pilares en PEEK. Las propiedades mecánicas del pilar en PEEK, por su bajo módulo de elasticidad, presentaron una deformación inicial en toda el área del pilar, transmitiendo esfuerzos al tornillo que se encuentra dentro del pilar plástico, en el momento 1.5 meses, los valores von Mises del tornillo en el pilar en PEEK subieron hasta $560 \mathrm{Mpa}$, acercándose al límite de fluencia de $800 \mathrm{Mpa}$ del titanio. Hugo Eduardo Peixoto et al. (8) evaluaron la influencia del material del pilar y la geometría en la distribución de esfuerzos en implantes unitarios. Tanto el pilar de titanio como el de circonio mostraron esfuerzos von Mises similares en todos los modelos; sin embargo, independiente del material del pilar, el tornillo fue la pieza más afectada.

Al comparar el modelo de pilar en titanio en el día cero con el del momento 1.5 meses, se observa que los esfuerzos del implante disminuyen a la mitad con la formación de hueso crestal, el contacto que se da del hueso crestal con el implante en el momento 1.5 meses hace que el sistema distribuya en una mayor área las fuerzas comparado con en el día cero, por otro lado, los modelos de pilares en PEEK, en la fase 1.5 y 3 meses, mostraron casi dos veces mayor concentración de fuerzas en el implante que en los sistemas con pilar en titanio. Roldán (14) y cols ${ }^{14}$ encontraron resultados similares, donde el pilar, el tornillo y el implante fueron los elementos que presentaron valores von Mises más elevados.

En la fase 1.5 y 3 meses, a pesar de tener diferentes valores de densidad ósea en la porción crestal, al comparase la distribución de los esfuerzos en todo el sistema, se observaron valores similares. 
Mauricio Alejandro Rodríguez López, Samuel David Giraldo Gómez, Federico Latorre Correa, 13 Junes Abdul Villarraga Ossa, Juan Gustavo Diosa Peña

De los resultados obtenidos se confirma la importancia de un hueso de buena densidad en la zona apical en el implante postexodoncia y el uso con precaución con pilares PEEK, como lo indica la casa comercial, debido a su menor resistencia y mayor deformación, que implicaría sobrecargas en otras áreas como el tornillo, el implante y el área de cicatrización ósea apical.

Este estudio es una simulación in vitro, presenta información teórica sobre el comportamiento de los elementos modelados, por eso no se debe extrapolar directamente a la parte clínica sin las investigaciones complementarias del caso.

\section{Conclusiones}

- El hecho que el implante se encuentre en contacto con el hueso solo en la porción apical y que el coágulo no aporte a la distribución de esfuerzos, hace que se aumente la concentración de esfuerzos en el tornillo, el implante y en el mismo hueso esponjoso apical.

- Por las características plásticas de los pilares en PEEK, se pueden encontrar valores importantes de esfuerzos en los tornillos del sistema. Factor que se debe tener en consideración por ser el tornillo un componente critico en los implantes.

- La aparición de hueso crestal y el contacto que se da con el implante en la fase 1.5 meses, hace que el implante pueda distribuir mejor los esfuerzos al cual está sometido desde el día cero.

- Los pilares en PEEK en la fase 1.5 y 3 meses trasmiten el doble de fuerzas al implante que los pilares en titanio.

\section{Conflicto de interes}

Los autores dan fe que no tienen ningún conflicto de interés en la elaboración de esta investigación.

\section{Referencias}

1. Jemt T, Lekholm U, Adell R. Osseointegrated implants in the treatment of partially edentulous patients: a preliminary study on 876 consecutively placed fixtures. Int J Oral Maxillofac Implants. 1989; 4: 211-217. 
Evaluación de la distribución de esfuerzos de una restauración inmediata implantosoportada en tres fases de cicatrización. Análisis de elementos finitos

2. Stanford CM. Application of oral implants to the general dental practice [published correction appears in JADA 2005; 136(10): 1372]. JADA 2005; 136(8): 1092-100.

3. Lekholm U, Zarb GA. Patient selection. Tissue-Integrated Prostheses: Osseointegration in Clinical Dentistry. Chicago: Quintessence Publishing; 1985. 199-209 p.

4. Brunski JB. Biomechanical factors affecting the bone- dental implant interface. Clinical Materials. 1992; 10: 153-201.

5. Wazen RM. Micromotion-induced strain fields influence early stages of repair at bone-implant interfaces. Acta Biomaterialia. 2013; 9: 6663-6674. doi: 10.1016/j.actbio.2013.01.014

6. Eskitascioglu G. The influence of occlusal loading location on stresses transferred to implantsupported prostheses and supporting bone: A three-dimensional finite element study. J Prosthet Dent. 2004; 91: 144-50. doi: 10.1016/\$0022391303007418

7. Caglar A. Three-Dimensional Finite Element Analysis of Titanium and Yttrium-Stabilized Zirconium Dioxide Abutments and Implants. Internacional Journal of Oral and Maxilofacial Implants. 2011; 26(5).

8. Peixoto HE. The role of prosthetic abutment material on the stress distribution in a maxillary single implant-supported fixed prosthesis. Materials Science and Engineering. 2016; 65(1): 90-96. doi: 10.1016/j.msec.2016.04.004

9. Rae PJ. The mechanical properties of poly (ether-ether-ketone) (PEEK) with emphasis on the large compressive strain response. Polymer. 2007; 48: 598e615.

10. Koutouzis T, Richardson J, Lundgren T. Comparative soft and hard tissue responses to titanium and polymer healing abutments. J Oral Implantology. 2011;37: 174-82. doi: 10.1563/ AAID-JOI-D-09-00102.1

11. Murakami N, Wakabayashi N. Finite element contact analysis as a critical technique in dental biomechanics: A review. J Prosthodont Research. 2014; 58:92-101. doi: 10.1016/j. jpor.2014.03.001

12. Trivedi S. Finite element analysis: A boon to dentistry. Journal of Oral Biology and Craniofacial Research. 2014; (4): 200-203.

13. Kurniawan D, Nor FM, Lee HY, Lim JY. Finite element analysis of bone-implant biomechanics: refinement through featuring various osseointegration conditions. Int J Oral Maxillofac Surg. 2011; 41(9): 1090-1096. doi: 10.1016/j.ijom.2011.12.026 
Mauricio Alejandro Rodríguez López, Samuel David Giraldo Gómez, Federico Latorre Correa, 15 Junes Abdul Villarraga Ossa, Juan Gustavo Diosa Peña

14. Vanegas-Acosta JC, Landinez PNS, Garzón Alvarado DA, Casale RMC. A finite element method approach for the mechanobiological modeling of the osseointegration of a dental implant. Computer methods and programs in biomedicine. 2011; 101: 297-314. doi: 10.1016/j. cmpb.2010.11.007

15. Sanz M, Vignoletti F. Immediate implants at fresh extraction sockets: from myth to reality. Periodontology 2000. 2014; 66: 132-152. doi: 10.1111/prd.12044

16. Roldán Valencia FM, Escobar Restrepo JC, Latorre Correa F. Análisis lineal y no lineal de los esfuerzos en pilares de circonio preformados. Elementos finitos. Revista Ingeniería Biomédica. 2014; 8(16): 39-50.

17. Alkan I, Sertgöz A, Ekici B. Influence of occlusal forces on stress distribution in preloaded dental implant screws. J Prosthodontic Dentistry. 2004; 91(4): 319-25.

18. A multi-attachment approach to partial dentures. Sterngold attachments. [Internet]. [Consultado 24 de junio de 2016]. doi: 10.1016/S0022391304000332

19. Schwitalla AD. Finite element analysis of the biomechanical effects of PEEK dental implants on the peri-implant bone. Journal of Biomechanics. 2015; 48: 1-7. doi: 10.1016/j. jbiomech.2014.11.017

20. Steven M, Kurtza B, John N, Devine. PeEk biomaterials in trauma, orthopedic, and spinal implants. Biomaterials. 2007; 28: 4845-4869.

21. Ridwan-Pramana A. Structural and mechanical implications of PMMA implant shape and interface geometry in cranioplasty. A finite element study. J Craniomaxillofac Surgery. 2016; (44): 34-44. doi: 10.1016/j.jcms.2015.10.014

22. Alkan I. Influence of occlusal forces on stress distribution in preloaded dental implant screws. J Prosthodontic Dentistry. 2004; 91(4): 319-25.

23. Sanz-Sánchez I, Sanz-Martín E, Figuero M, Sanz M. Clinical efficacy of immediate implant loading protocols compared to conventional loading depending on the type of the restoration: a systematic review. Clin Oral Implants Res. 2014; 26(8): 964-82. doi: 10.1111/clr.12428

24. Esposito M. Interventions for replacing missing teeth: different times for loading dental implants. Cochrane Database Syst Rev. 2013; 3(1). doi: 10.1002/14651858.CD003878.pub5

25. NeumannEA. Fracture resistance ofabutmentscrews made of titanium, polyetheretherketone, and carbon fiber-reinforced polyetheretherketone. Braz Oral Res. 2014; 28(1): 1-5. 